\title{
Rapid determination of cysteine and chiral discrimination of D-/L-cysteine via the aggregation-induced emission enhancement of gold nanoclusters by $\mathrm{Ag}^{+}$
}

Shengli RUAN,* Yan ZHOU,** Min ZHANG,**广 Hongyang ZHANG,* Yuerong WANG,* Ping $\mathrm{HU}^{* \dagger}$

* Shanghai Key Laboratory of Functional Materials Chemistry, School of Chemistry and Molecular Engineering, East China University of Science and Technology, Shanghai 200237, China

** Engineering Research Centre of Pharmaceutical Process Chemistry, Ministry of Education, \& Shanghai Key Laboratory of New Drug Design, School of PharmacyDepartment, East China University of Science and Technology, Shanghai 200237, China

To whom correspondence should be addressed.

E-mail: huping@ecust.edu.cn, zhangm@ecust.edu.cn 


\begin{abstract}
Cysteine (Cys) plays vital roles in various physiological and pathological functions. Either deficiency or excess of Cys could lead to severe ailments in human. Identification and determination of Cys are the key issues for the early diagnosis of relevant diseases. This contribution presented a promising potential of fluorescent gold nanoclusters (AuNCs) for Cys determination and D-/L-Cys enantiomer discrimination. Cys determination and discrimination involved three steps. First, as a reducing and capping ligand, glutathione was applied to fabricate weak fluorescent AuNCs. Second, $\mathrm{Ag}^{+}$was introduced to lead the aggregation-induced emission (AIE) to form well-dispersed aggregates. The fluorescence intensity of AuNCs was monitored at excitation/ emission wavelengths of 396/620 nm. Third, Cys was found to quickly bind with $\mathrm{Ag}^{+}$to form a grid network to light up the system via aggregation-induced emission enhancement (AIEE). A novel sensor for sensitive and visually selective detection of Cys was established on the basis of AIEE mechanism. Rapid quantitative determination of Cys was achieved in two minutes via AIEE within the range of $0.5-100 \mu \mathrm{mol} \mathrm{L}^{-1}$ and a detection limit of $0.365 \mu \mathrm{mol} \mathrm{L} \mathrm{L}^{-1}$. Moreover, due to the specific interactions of D-/L-Cys with mandelic acid and tartaric acid, visual discrimination of D-/L-Cys enantiomers with naked eyes was realized by replacing the organic acid buffer.
\end{abstract}




\section{Introduction}

Cysteine (Cys) is a thio-containing amino acid that widely exists in different organisms and controls the three-dimensional structure of proteins through the formation of disulfide bonds. Cys plays vital roles in various physiological and pathological functions, such as detoxification ${ }^{1}$ and metabolism. ${ }^{2,3}$ The deficiency of Cys could lead to decreased hematopoietic function, ${ }^{4}$ edema, ${ }^{5}$ psoriasis, ${ }^{6}$ growth retardation, ${ }^{7,8}$ while liver damage, ${ }^{9}$ and excessive Cys could resulted in neurodegenerative diseases, ${ }^{10}$ amyotrophic lateral sclerosis, and Huntington's disease. ${ }^{11}$ Therefore, accurate identification and determination of Cys are the key issues for the early diagnosis of relevant diseases.

The enantiomers of Cys also have different contrasting effects on the physical function of humans. Previous studies have shown that L-Cys at a low level acted as a neuroprotective antioxidant in neuronal activity, while a high level of L-Cys could cause neuronal damage. ${ }^{12}$ By contrast, D-Cys caused no excitotoxic damage to the brain and protected cerebellar neurons more effectively than L-Cys. The result relied on the higher transport activity of D-Cys than that of L-Cys at the same concentration. ${ }^{13,14}$ Therefore, the selective recognition of Cys enantiomers has been focused within biochemistry and chiral drug regimes. In this scenario, developing a more efficient sensor for stereoselective analysis of Cys enantiomer is urgently needed. Various analytical techniques, including mass spectrometry, high performance liquid chromatography, capillary electrophoresis, and other electrochemistry techniques, have been applied to determine Cys. However, these methods require expensive equipment. Using fluorescent sensors, by contrast, is quite a simple method to perform Cys determination.

Gold nanoclusters (AuNCs) have drawn much attention for their fundamental properties and practical applications. ${ }^{15}$ As a new type of fluorescent nanomaterial, 
AuNCs have expressed remarkable potentials in sensing because of their distinctive features of superior biocompatibility and tunable fluorescence emission within the visible to the near-infrared range. The preparation of AuNCs usually starts from reduction of $\mathrm{Au}(\mathrm{III})$ and ends with formation of atomic $\mathrm{Au}(0) .{ }^{16}$ The growth mechanism has been fully elucidated by Xie et al. ${ }^{17}$ The intermediate $\mathrm{Au}(\mathrm{I})$ also showed potential advantages in sensing and recognition of specific targets attributed to the fluorescence tuning of $\mathrm{Au}(\mathrm{I})$ complexes. ${ }^{18}$ These complexes were prepared with the addition of reducing agent and stabilizing agent. Water-soluble biothiols were popularized in fabricating AuNCs for the formation of Au-S bond because of gold-thiol interactions. ${ }^{19}$ Glutathione (GSH) is the most common candidate as a reducing and capping ligand to obtain AuNCs. ${ }^{20}$

Aggregation-induced emission (AIE), which was discovered by Tang et al., was first reported in organic molecules regime. ${ }^{21}$ Fluorophores generally show bright fluorescence in dilute solutions but weak fluorescence in concentrated solutions or agglomerations with aggregation-caused quenching (ACQ) effect. The ACQ effect results in drawbacks of weak resistance to photobleaching, high background, and poor photosensitivity. The AIE and AIE enhancement (AIEE) characteristics of various types of fluorophores have been found to have completely opposite effects on ACQ. These AIE luminescence agents (AIEgens) usually show negligible fluorescence in dilute solutions but emit strong fluorescence when forming aggregates. The properties of the AIEgens were attractive in fields of biological detection and solid-state optical materials or devices fabrication. In recent reports, novel metal nanoclusters also expressed AIE properties. Beneficial luminescent nanoclusters, such as $\mathrm{Au},{ }^{22-24} \mathrm{Cu},{ }^{25}$ and $\mathrm{Ag},{ }^{26}$ have been extensively focused on, of which AuNCs have been the frontrunner due to their high stabilities. ${ }^{27}$ AIE has played a major role in the luminescence properties of nanoclusters, where the aggregation degree acts as a focal point for emission 
wavelength and the intensity. The AIE in NCs occurs through self-assembled routes (non-electrostatic and electrostatic), which usually results in considerable blueshifts in emission accompanied by the transformation from a disordered to an ordered morphology.

In this contribution, the AIEE properties of AuNCs were applied to sensitively and rapidly determine Cys. The sensing strategy is described in Scheme 1. In the presence of $\mathrm{Ag}^{+}$, the coordination of the carboxyl group of AuNCs at the GSH terminal and $\mathrm{Ag}^{+}$ shortened the distance between AuNCs and improved the fluorescence emission of AuNCs. As a bridge, $\mathrm{Ag}^{+}$interacting with GSH-AuNCs through coordination connected with Cys to form an aggregated network. The aggregation state resulting in AIEE occurrence greatly enhanced the fluorescence emission of AuNCs. The level of Cys could also be quantitatively determined depending on the increase in fluorescence intensity. As AIEE occurred very rapidly, the detection process could be completed within two minutes. Moreover, due to the specific interactions of D-/L-Cys with mandelic acid and tartaric acid, the chiral discrimination of D-/L-Cys with naked eyes was realized by replacing the organic acid buffer.

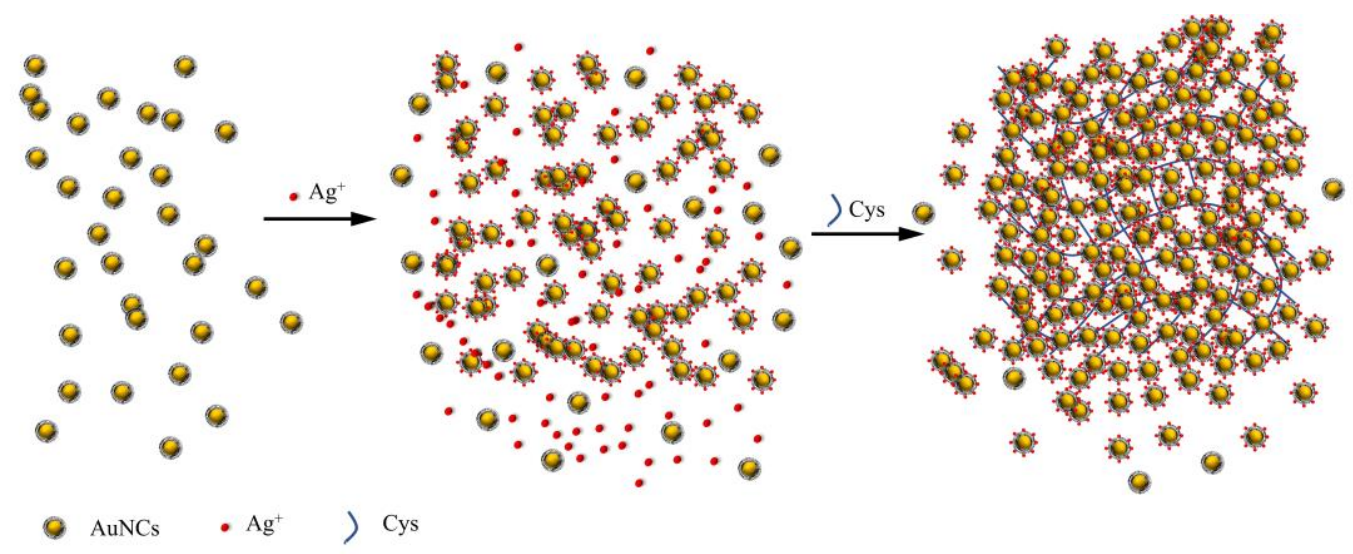

Scheme 1. Schematic of cysteine determination via aggregation-induced emission of AuNCs induced by $\mathrm{Ag}^{+}$ 


\section{Experimental}

Reagents and chemicals

Silver nitrate $\left(\mathrm{AgNO}_{3}\right)$, glutathione (GSH), hydrogen tetrachloroaurate (III) trihydrate $\left(\mathrm{HAuCl}_{4} \cdot 3 \mathrm{H}_{2} \mathrm{O}\right)$ and amino acids were purchased from Adamas-Beta Co., Ltd. Other inorganic reagents were obtained from Shanghai Lingfeng Chemical Reagent Co., Ltd. without further purification. The water applied in all experiments was deionized (resistivity $>18 \mathrm{M} \Omega \mathrm{cm}-1$ ).

\section{Preparation of GSH-AuNCs}

Typically, freshly prepared aqueous solution of $\mathrm{HAuCl}_{4}(20 \mathrm{mM}, 5 \mathrm{~mL})$ and $\mathrm{GSH}$ $(100 \mathrm{mM}, 2.5 \mathrm{~mL})$ were mixed with $43.5 \mathrm{~mL}$ of deionized water under gentle stirring at $25^{\circ} \mathrm{C}$ for $5 \mathrm{~min}$. The precipitate was observed, and then aqueous solution of $\mathrm{NaOH}(1.0$ M) was added to the mixture to adjust the $\mathrm{pH}$ to $\sim 7.0$. After the precipitate was dissolved, the reaction mixture was heated to $90{ }^{\circ} \mathrm{C}$ under gentle stirring for $24 \mathrm{~h}$. Eventually the obtained light yellow aqueous solution was dialyzed using a dialysis bag (MWCO: $2000 \mathrm{Da}$ ) against deionized water for $24 \mathrm{~h}$. The AuNCs were stored at $4{ }^{\circ} \mathrm{C}$ before use.

\section{Transitional metal ions induced AIEE}

AIEE of GSH-AuNCs was induced by transition metal ions including $\mathrm{Ag}^{+}, \mathrm{Cr}^{3+}$, $\mathrm{Cd}^{2+}, \mathrm{Ce}^{3+}, \mathrm{Co}^{2+}, \mathrm{Cu}^{2+}, \mathrm{Pb}^{2+}, \mathrm{Zn}^{2+}, \mathrm{Fe}^{2+}$, and $\mathrm{Fe}^{3+}$. The concentration of metal ions was kept at $10 \mathrm{mM}$. The variation of fluorescence intensity was recorded to investigate the coordination between metal ions and GSH-AuNCs. The fluorescence spectrum was measured by a spectrometer. 
Determination of L-Cys based on $\mathrm{Ag}^{+}$induced AIEE

A volume of $500 \mu \mathrm{L}$ of freshly prepared citrate buffer $(0.1 \mathrm{M}, \mathrm{pH} 4.0)$ containing 0-10 $\mathrm{mM} \mathrm{Ag}^{+}$was mixed with the AuNCs complex $(1: 1, \mathrm{v} / \mathrm{v})$. The fluorescence emission spectra were recorded in the range of 500-800 nm with an excitation wavelength of $396 \mathrm{~nm}$. A volume of $500 \mu \mathrm{L}$ of freshly prepared citrate buffer $(0.1 \mathrm{M}$, $\mathrm{pH}$ 4.0) containing 0-100 $\mu \mathrm{M}$ L-Cys was mixed with the AuNCs-Ag ${ }^{+}$complex (1:1, v/v). The fluorescence emission spectra measurement carried out by spectra was recorded in the range of 500-800 nm with an excitation wavelength of $396 \mathrm{~nm}$.

\section{Determination of L-Cys in human serum samples}

Human serum samples were from healthy individual at Jiangxi Provincial People's Hospital. The L-Cys solutions at different concentrations of $1.0 \mu \mathrm{M}, 10 \mu \mathrm{M}$, and $50 \mu \mathrm{M}$ were then spiked into the diluted samples and further added into the GSH-AuNCs-Ag+ sensing system. The measurements were carried out after intensive blending for $0.5 \mathrm{~min}$. The concentration of Cys in serum was determined by the method established in this work.

Determination of L-Cys in human serum samples

A volume of $500 \mu \mathrm{L}$ of freshly prepared tartaric acid/ mandelic acid $(0.1 \mathrm{M})$ containing D/L-Cys was mixed with the AuNCs and $\mathrm{Ag}^{+}$complex (1:1, v/v). Photos of samples under sunlight and ultraviolet light $(365 \mathrm{~nm})$ were recorded and UV spectrum was collected. The fluorescence emission spectra were recorded in the range of 500-800 $\mathrm{nm}$ with an excitation wavelength of $396 \mathrm{~nm}$.

\section{Apparatus}

Fluorescence spectra were recorded on an F-4600 spectrofluorophotometer 
(HITACHI Co., Ltd., Japan). UV-vis absorption spectra were monitored on a UV-2550 UV-Vis spectrometer (Shimadzu Co., Ltd.). Transmission electron microscopy (TEM) was measured on a JEM-2100 high resolution transmission electron microscope (HITACHI Co., Ltd., Japan). Dynamic light scattering (DLS) measurements were conducted Malvern Zetasizer Nano ZSE (Malvern Panalytical Ltd).

\section{Results and Discussion}

\section{Characterization of GSH-AuNCs}

The optical behavior of GSH-AuNCs was measured using a UV-Vis absorption spectrometer and fluorescence spectrometer. Fig. 1 shows the UV-Vis spectrum of the AuNCs prepared under neutral conditions. In this work, the GSH molecules acted as a reducing agent and a template. As shown in Fig. 1, the fluorescence emission peak of AuNCs prepared at neutral condition was at $620 \mathrm{~nm}$, with the excitation at $396 \mathrm{~nm}$. The morphologies of GSH-AuNCs were characterized by TEM. As shown in Fig. 2(A), the GSH-AuNCs prepared in this work were spherical and in uniform size, which has a distinct lattice structure. The results of characterization and optical properties of GSH-AuNCs are consistent with the previous synthesis protocols, indicating that AuNCs were successfully prepared..$^{28}$



Fig. $1 \mathrm{UV}-$ Vis spectrum (blue trace) and fluorescence emission spectrum (red trace) of GSH-AuNCs 

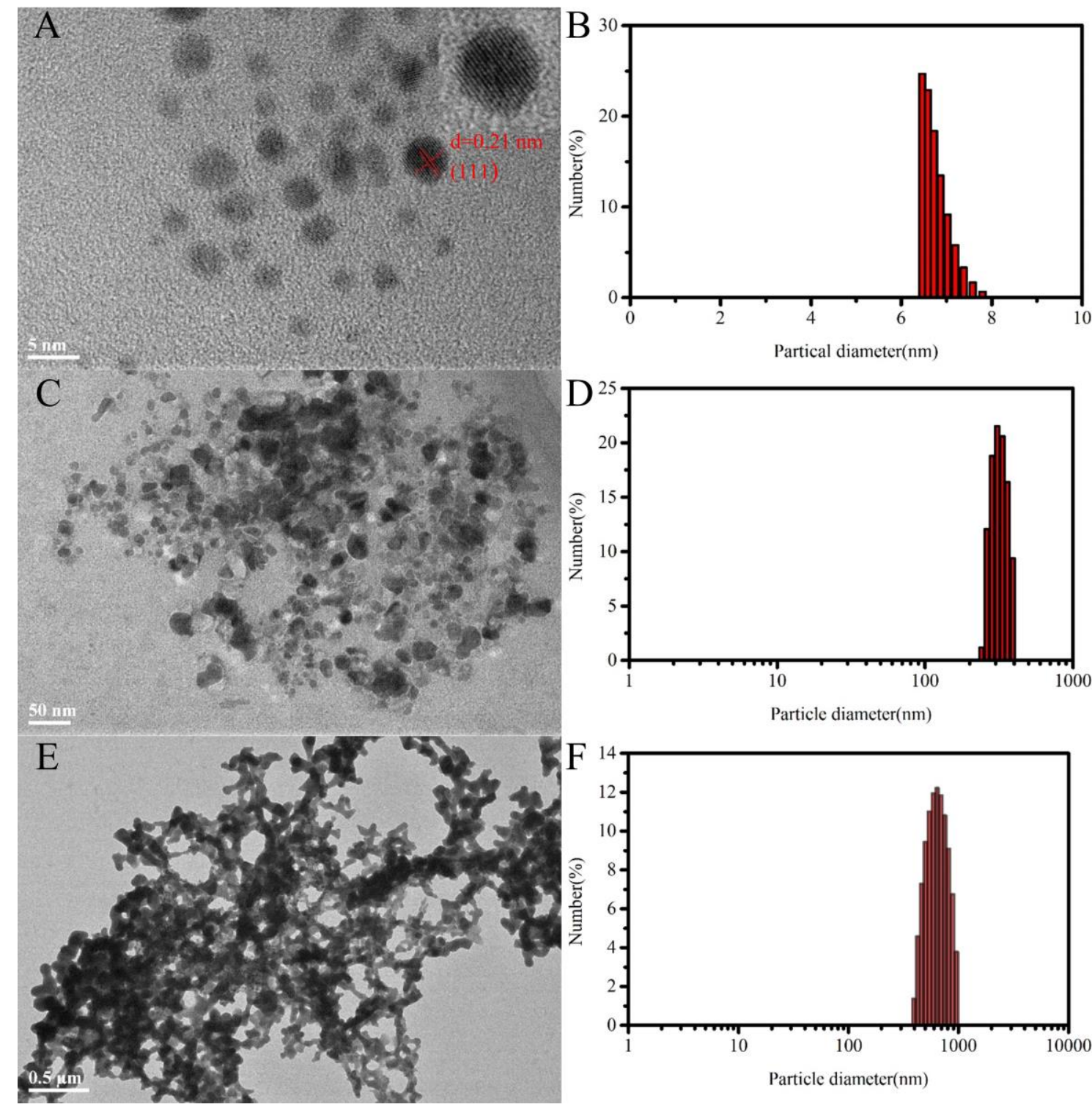

Fig. 2 TEM images of GSH-AuNCs with a dimension of (A) $5 \mathrm{~nm}$ (Inset: lattice spacing image), (C) $50 \mathrm{~nm}$, and (E) $0.5 \mu \mathrm{m}$ and the corresponding particle distribution and the DLS spectra of (B) GSH-AuNCs, (D) GSH-AuNCs/Ag+, and (F) GSH-AuNCs/ $\mathrm{Ag}^{+} / \mathrm{L}-\mathrm{Cys}$ system, the concentrations of $\mathrm{Ag}^{+}$and L-Cys was $7.5 \mathrm{mM}$ and $50 \mu \mathrm{M}$, respectively. 


\section{Coordination between GSH-AuNCs and transitional metal ions}

The GSH molecule rich in sulfhydryl moiety (-SH) and coordinative atoms is able to interact with metal ions. As GSH coordinated with positive-charged metal ions, coordination bonds were formed between the lone pair electrons of GSH and the unoccupied orbit of metal ions. ${ }^{29}$ In the present work, the interaction between transitional metal ions and GSH-AuNCs was probed by investigating the fluorescence intensity variation. Fig. 3(C) shows the fluorescence intensity variation of GSH-AuNCs by the addition of various transition metal ions. The fluorescence spectrometry results showed that all the selected transitional metal ions $\left(\mathrm{Ag}^{+}, \mathrm{Cr}^{3+}, \mathrm{Cd}^{2+}, \mathrm{Ce}^{3+}, \mathrm{Co}^{2+}, \mathrm{Cu}^{2+}\right.$, $\mathrm{Pb}^{2+}, \mathrm{Zn}^{2+}, \mathrm{Fe}^{2+}$, and $\mathrm{Fe}^{3+}$ ) could coordinate with GSH-AuNCs. The process realized the charge transfer between the ligand molecule and the metal ion and aroused fluorescence changes in GSH-AuNCs. As shown in Fig. 3(B), the metal ions of $\mathrm{Ag}^{+}, \mathrm{Cd}^{2+}, \mathrm{Ce}^{3+}, \mathrm{Pb}^{2+}$, and $\mathrm{Zn}^{2+}$ noticeably formed visible coordination with GSH-AuNCs, and significant fluorescence enhancement was observed. Similar visible complexes also formed between $\mathrm{Cu}^{2+}$ and GSH-AuNCs, while the fluorescence of the complex was quenched. This phenomenon possibly attributed to the relatively less charge transfer of the ions $\left(\mathrm{Ag}^{+}, \mathrm{Cd}^{2+}, \mathrm{Ce}^{3+}, \mathrm{Pb}^{2+}\right.$, and $\left.\mathrm{Zn}^{2+}\right)$ with $\mathrm{d} 10$ valence electron structure, and the charge easily transferred to the higher s-orbital. Compared with these ions, the charge transfer between $\mathrm{Cu}^{2+}$ and GSH was sufficient to increase the tendency of oxidizing GSH, and $\mathrm{Cu}^{2+}$ was found to be already being reduced to $\mathrm{Cu}^{+}{ }^{30}$ The metal ions of $\mathrm{Co}^{2+}, \mathrm{Fe}^{2+}, \mathrm{Fe}^{3+}$, and $\mathrm{Cr}^{3+}$ could also coordinate with GSH-AuNCs, but the complex had higher solubility in water, leading to fluorescence quenching. 

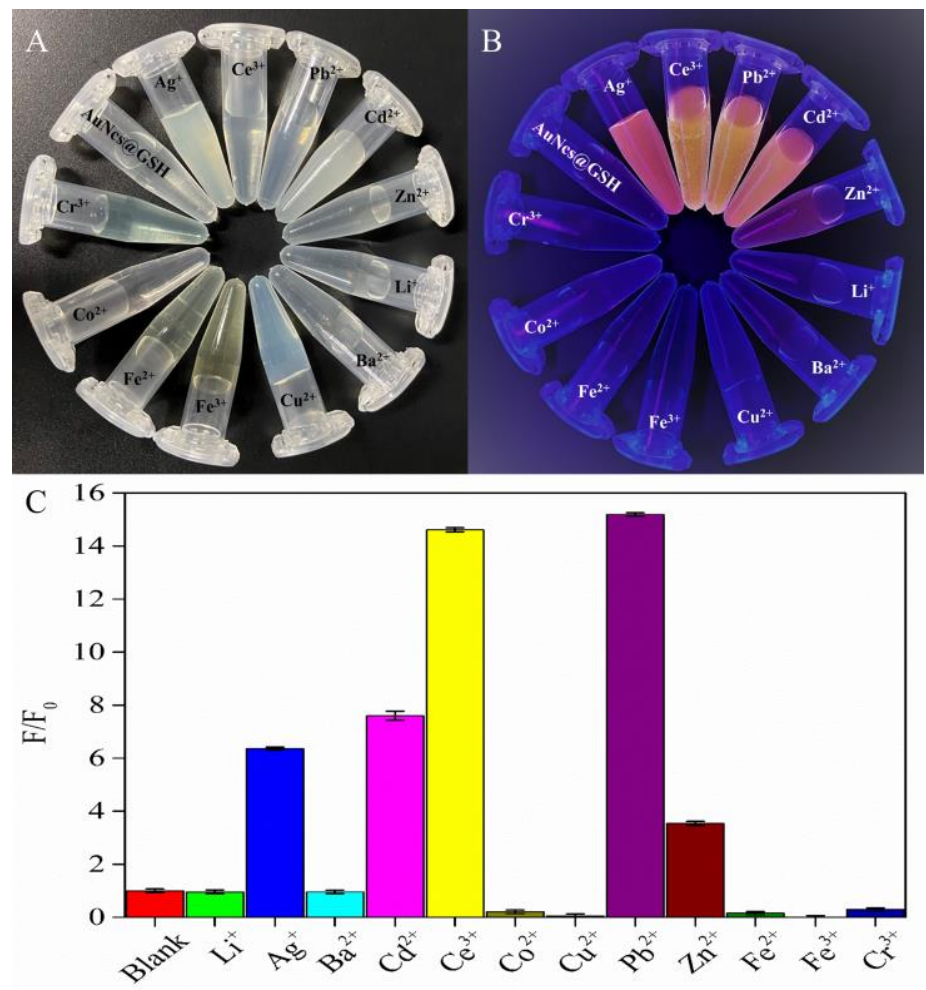

Fig. 3 Digital photographs of GSH-AuNCs responding to 12 ions under (A) visible light and (B) UV light. (C) Luminescence intensities at $620 \mathrm{~nm}$ of aqueous GSH-AuNCs solutions in the presence of different metal ions.

Since $\mathrm{pH}$ could affect the stability of GSH, citrate buffer ( $\mathrm{pH} 4.0$ ) was then added to form the association with GSH to prevent the decomposition of it. ${ }^{31}$ The effect of buffer solution on the coordination between GSH-AuNCs and ions was further investigated. As shown in Fig. 4(A) and (B), the addition of $\mathrm{Ag}^{+}, \mathrm{Cu}^{2+}, \mathrm{Fe}^{2+}$, and $\mathrm{Fe}^{3+}$ into AuNCs with the addition of citrate resulting in no obvious visual change while the fluorescence intensities of AuNCs varied differently. The fluorescence intensity of AuNCs induced by $\mathrm{Ag}^{+}$reached to over 6.5 -fold of its original level with the addition of citrate, while those by $\mathrm{Cd}^{2+}, \mathrm{Ce}^{3+}, \mathrm{Pb}^{2+}$, and $\mathrm{Zn}^{2+}$ were decreased to almost the same levels as the blank control, indicating a reversible coordination occurred between the ions and $\mathrm{GSH}-\mathrm{AuNCs}$ at $\mathrm{pH}=4.0$. The equilibrium was reached within a few seconds. This rapid enhancement of fluorescence under mild conditions revealed that the complex was formed. The coordination of the carboxyl group of AuNCs at the GSH 
terminal and $\mathrm{Ag}^{+}$shortened the distance between AuNCs even generate precipitate and improved the fluorescence emission of AuNCs. Therefore, citrate was introduced to induce the aggregation of AuNCs by $\mathrm{Ag}^{+}$.
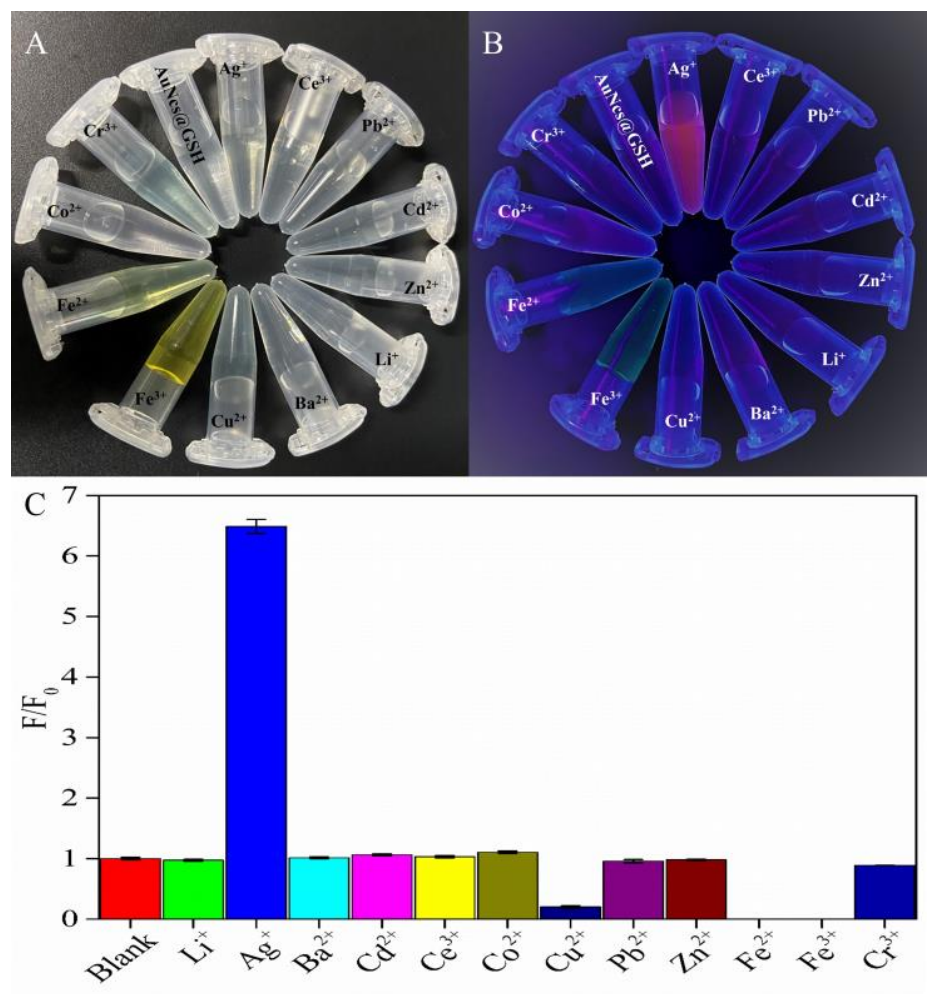

Fig. 4 Digital photographs of GSH-AuNCs responding to 12 ions in the citrate solution ( $\mathrm{pH}=4,100 \mathrm{mM})$ under (A) visible light and (B) UV light. (C) Luminescence intensities at $620 \mathrm{~nm}$ of aqueous GSH-AuNCs solutions in the presence of different metal ions. $\mathrm{F}_{0}$ and $\mathrm{F}$ are the fluorescence intensity of GSH-AuNCs in the absence and presence of different metal ions, respectively.

Determination of L-Cys by $\mathrm{Ag}^{+}$triggered AIEE of GSH-AuNCs

As shown in Fig. 5, the fluorescence intensity of GSH-AuNCs was increased 5.37-fold with the addition of $\mathrm{Ag}^{+}$. The photo insets displayed more visualized evidence. The red fluorescence of GSH-AuNCs/ $\mathrm{Ag}^{+}$solution was significantly stronger than that of GSH-AuNCs. Figure 2(C) showed the TEM images of GSH-AuNCs/ $\mathrm{Ag}^{+}$complex. With the introduction of $\mathrm{Ag}^{+}$, the well-dispersed GSH-AuNCs (Fig. (2A)) were 
extensively aggregated. As mentioned above, $\mathrm{Ag}^{+}$could coordinate with the GSH-AuNCs which caused the GSH-AuNCs to aggregate. The enhanced fluorescence of GSH-AuNCs caused by the addition of $\mathrm{Ag}^{+}$could be attributed to the AIEE effect.



Fig. 5 The fluorescence spectra of GSH-AuNCs (black curve), GSH-AuNCs/L-Cys (red curve), GSH-AuNCs/Ag ${ }^{+}$(blue curve) and GSH-AuNCs/ $\mathrm{Ag}^{+} / \mathrm{L}-\mathrm{Cys}$ (pink curve). Photo insets, (A) GSH-AuNCs/L-Cys, (B) GSH-AuNCs/Ag ${ }^{+}$, and (C) GSH-AuNCs/Ag $/$ L-Cys.

After Cys was added, the fluorescence intensity of the AuNCs/Ag ${ }^{+}$system increased 6.44-fold, whereas that of GSH-AuNCs had no obvious variation. The result illustrated that the $\mathrm{Ag}^{+}$-triggered "light-up" GSH-AuNC sensing system was competent for the detection of Cys. The DLS data in Fig. 2(F) showed that the addition of Cys further increased the aggregation degree of the GSH-AuNCs/Ag ${ }^{+}$system. Moreover, a grid network (Fig. 2(E)) that was formed after the addition of Cys resulted in fluorescence enhancement as Cys exhibited strong binding ability for metal ions and formed C-metal ion-C base pairs. ${ }^{32}$ As a bridge, $\mathrm{Ag}^{+}$could interact with GSH-AuNCs through coordination and connect with Cys to form a grid network which could strengthen the AIEE effect. Therefore, the fluorescence intensity of the system could be greatly enhanced. Thus, the concentration of $\mathrm{Ag}^{+}$had an influence on the enhanced performance of $\mathrm{Ag}^{+}$-modulated "light-up" method.

The fluorescence intensity of the GSH-AuNCs system mixed with various 
concentrations of $\mathrm{Ag}^{+}$in the presence and absence of Cys is shown in Fig. S1 The optimized value of the fluorescence enhancement ratio between the GSH-AuNCs/ $\mathrm{Ag}^{+} / \mathrm{Cys}$ system and the GSH-AuNCs/Ag ${ }^{+}$system was obtained when the concentration of $\mathrm{Ag}^{+}$reached $7.5 \mathrm{mM}$. Therefore, $7.5 \mathrm{mM}$ of $\mathrm{Ag}^{+}$was chosen to realize the detection of Cys. Then, we investigated the effect of the mixed time. Fig. S2-(A) and Fig. S2-(B) showed the fluorescence intensities of $\mathrm{GSH}-\mathrm{AuNCs} / \mathrm{Ag}^{+}$system in the presence and absence of Cys in different mixed duration, respectively. The fluorescence intensity of the GSH-AuNCs/Ag ${ }^{+}$system reached peak within one minute with or without the addition of Cys. Thus, the whole determination process of Cys could be completed in two minutes.

The effect of $\mathrm{pH}$ on the fluorescence intensity of the $\mathrm{GSH}-\mathrm{AuNCs} / \mathrm{Ag}^{+} / \mathrm{Cys}$ system was further explored. GSH has four ionizable functional groups with pKa 2.12, 3.53, 8.66, and 9.12, respectively. ${ }^{33}$ The ionization equilibrium of GSH in solution affected the coordination between $\mathrm{Ag}^{+}$and GSH. Meanwhile, the GSH structure itself has $\mathrm{O}, \mathrm{N}$, and $\mathrm{S}$ electronegative atoms to bind with metal ions. As shown in Fig. 6, the solutions displayed low fluorescence intensity at strong acidic $\mathrm{pH}$ (2-3). In the strong acidic environment, the amino groups of GSH-AuNCs were protonated to generate a low interaction with $\mathrm{Ag}^{+}$. At $\mathrm{pH}=4$, the coordination between $\mathrm{GSH}$ and $\mathrm{Ag}^{+}$resulted in the strongest fluorescence emission. The fluorescence quenched by the increase in $\mathrm{pH}$ until $\mathrm{pH}=6$. As the $\mathrm{pH}$ of the solution increased, the sulfhydryl group in GSH became more electronegative and the enhanced coordination with $\mathrm{Ag}^{+}$caused the fluorescence of the solution enhanced at $\mathrm{pH}=7$. We speculate the reason for the above trend of $\mathrm{pH}$ effect on fluorescence enhancement was that the interaction between Cys and GSH-AuNCs $/ \mathrm{Ag}^{+}$was dominated by electronegative atoms and $\mathrm{pH}$ environment of solution. We then optimized the $\mathrm{pH}=4$ was the detection condition of Cys. 


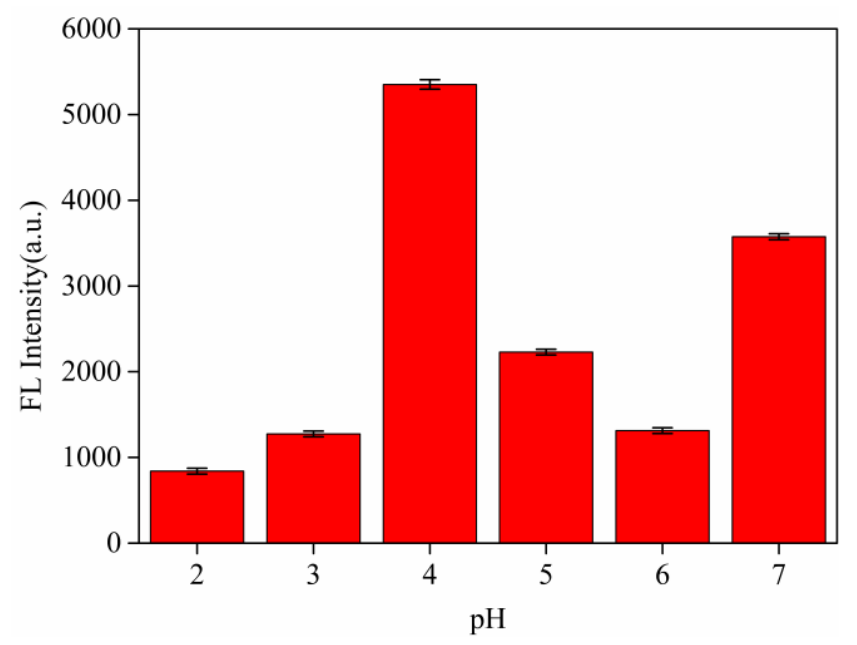

Fig. 6 Effect of $\mathrm{pH}$ on the fluorescence intensity of the GSH-AuNCs/ $\mathrm{Ag}^{+} / \mathrm{Cys}$ system.

Under the optimal conditions, various concentrations of Cys were mixed with GSH-AuNC/Ag ${ }^{+}$system. Meanwhile, the spectra were recorded in Fig. 7(A). With the increase of the Cys concentration from $0.5 \mu \mathrm{M}$ to $100 \mu \mathrm{M}$, the fluorescence intensity of the GSH-AuNC/Ag ${ }^{+}$sensing system gradually increased. As shown in Fig. 7(B), it was found that there was a linear relationship between the fluorescence intensity ratio $\left(\mathrm{F}-\mathrm{F}_{0}\right) / \mathrm{F}_{0}$ and the concentration of Cys, where $\mathrm{F}_{0}$ and $\mathrm{F}$ are the fluorescence intensity of GSH-AuNCs/ $\mathrm{Ag}^{+}$system in the absence and presence of Cys, respectively. The linear equation was $y=0.0659 x+0.0207$, with $R^{2}=0.993$. The detection limit was calculated to be $0.365 \mu \mathrm{M}$.
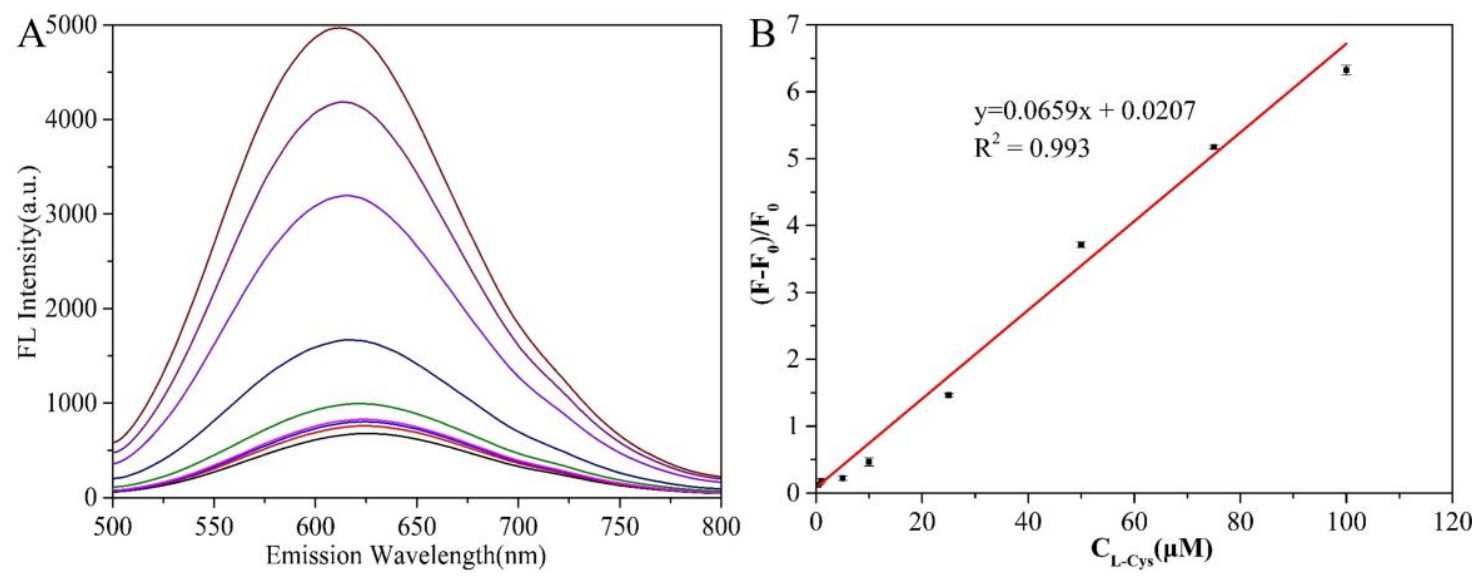

Fig. 7 (A) The fluorescence spectra of the GSH-AuNCs/ $\mathrm{Ag}^{+} / \mathrm{Cys}$ system mixed with 
different concentrations of L-Cys (from bottom to up were 0, 0.5, 1.0, 5.0, 10, 25, 50, 75, $100 \mu \mathrm{M}$. The fluorescence spectra were recorded in the 500-800 $\mathrm{nm}$ emission wavelength range at the excitation wavelength of $396 \mathrm{~nm}$. (B) The linearity of the fluorescence intensity ratio $\left(\mathrm{F}-\mathrm{F}_{0}\right) / \mathrm{F}_{0}$ versus value of L-Cys concentration. $\mathrm{F}_{0}$ and $\mathrm{F}$ are the fluorescence intensity of GSH-AuNCs/ $\mathrm{Ag}^{+}$system in the absence and presence of Cys, respectively.

\section{Specificity study}

The selectivity of AuNCs-based method for Cys determination was investigated. The fluorescence responses of $\mathrm{Ag}^{+}$-modulated GSH-AuNCs with the addition of Cys, homocysteine (Hcy), aspartic acid (Asp), histidine (His), isoleucine (Ile), alanine (Ala), glutamic acid (Glu), and lysine (Lys) were recorded. Fig. 8 shows only D-/L-Cys and Hcy resulted in significant fluorescence enhancement while other interfering amino acids had no obvious increase. Discrimination of Cys from Hcy could not be achieved by this method. In the case of GSH, the addition could not result in the fluorescence variation of GSH-AuNCs, thus improving the specificity of detection. The possible reason could be attributed to the steric hindrance of GSH molecule which could not cause visible aggregation of GSH-AuNCs. Hence, the above results exposed that the method based on $\mathrm{Ag}^{+}$-modulated GSH-AuNCs expressed high specificity for Cys recognition from interfering amino acids. 


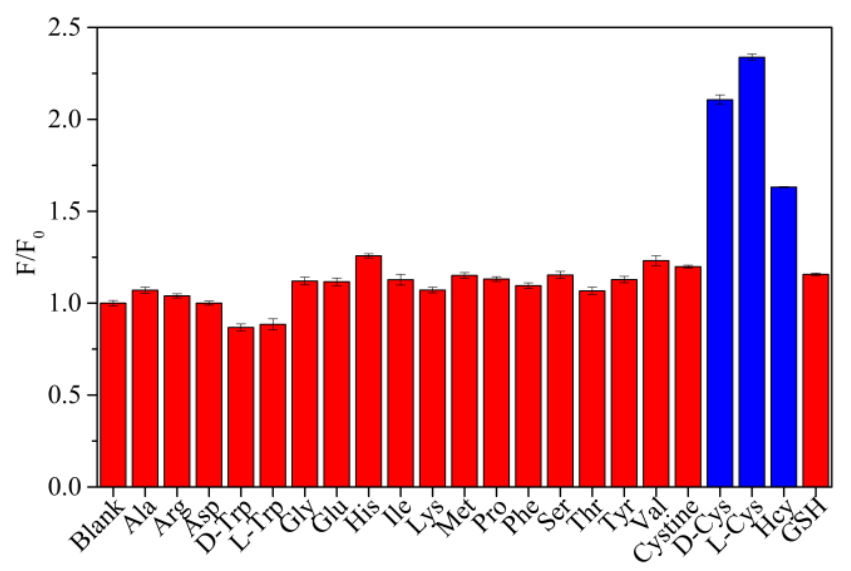

Fig. 8 The fluorescence intensity of the GSH-AuNCs/ $\mathrm{Ag}^{+}$system with the interfering amino acids, including alanine (Ala), arginine (Arg), aspartic acid (Asp), D-tryptophan (D-Trp), L-tryptophan (L-Trp), glycine (Gly), glutamic acid (Glu), histidine (His), isoleucine (Ile), lysine (Lys), methionine (Met), proline (Pro), phenylalanine (Phe), serine (Ser), threonine (Thr), tyrosine (Tyr), valine (Val), cysteine, D-Cys, L-Cys, homocysteine (Hcy), GSH. Concentration: $\mathrm{Ag}^{+}, 7.5 \mathrm{mM}$; cysteine , D/L-Cys, Hcy, GSH $25 \mu \mathrm{M}$; other amino acids, $1000 \mu \mathrm{M}$.

\section{Determination of L-Cys in human serum}

The L-Cys in diluted human serum samples was investigated to further evaluate the GSH-AuNCs application in the samples. Standard addition method was applied to measure the recoveries of Cys in the serum samples. Different concentrations of Cys were spiked to the samples. As shown in Table 1, the GSH-AuNC sensor showed reasonable recoveries in the range of $106.7 \%-111.3 \%$ and exhibited good reproducibility, with RSDs of less than $7.7 \%$. The obtained recoveries of Cys detection were satisfactory, indicating that this proposed fluorescence AIEE method has good feasibility for measuring Cys in human serum matrices. 
Table 1 The results of determination of L-Cys in human serum

\begin{tabular}{ccccc}
\hline Sample & Added $(\mu \mathrm{M})$ & Found $(\mu \mathrm{M})$ & Recovery $(\%$, & RSD $(\%, \mathrm{n}=6)$ \\
\hline \multirow{3}{*}{ Human serum } & 1.0 & $1.11 \pm 0.09$ & 111.3 & 7.7 \\
& 10.0 & $10.30 \pm 0.27$ & 103.0 & 2.6 \\
& 50.0 & $53.35 \pm 2.13$ & 106.7 & 0.4 \\
\hline
\end{tabular}

\section{Comparison of different analytical methods}

The method developed in this study was for the rapid determination of Cys. Various probes have already been reported for the analysis of Cys in different samples. Table 2 shows the comparative results for the different methods. The method used in this work has the advantage of detection time. This advantage has prospects in the rapid determination of Cys.

Table 2 Comparison of various fluorescent probes applications for the determination of Cys

\begin{tabular}{|c|c|c|c|c|}
\hline Method & System & $\begin{array}{c}\text { Linear } \\
\text { range }(\mu \mathrm{M})\end{array}$ & $\begin{array}{c}\text { Detection } \\
\text { limit }(\mu \mathrm{M}) \\
\end{array}$ & $\begin{array}{c}\text { Detection } \\
\text { Time(min) } \\
\end{array}$ \\
\hline Method in this paper & GSH-AuNCs-Ag ${ }^{+}$ & $0.5-100$ & 0.365 & 2 \\
\hline Method $\mathrm{A}^{27}$ & GSH-AuNCs-Ce $\mathrm{C}^{3+}$ & $0.4-120$ & 0.15 & 30 \\
\hline Method B ${ }^{34}$ & $\begin{array}{l}\text { chemical probe } \\
\text { (CR1) }\end{array}$ & $0-20$ & 0.029 & 60 \\
\hline Method $C^{35}$ & $\begin{array}{l}\text { chemical probe } \\
(\text { Сy.7-PT })\end{array}$ & $0-100$ & 0.39 & l \\
\hline Method $\mathrm{D}^{36}$ & $\begin{array}{l}\text { chemical probe } \\
\text { (Cys-AN) }\end{array}$ & l & 0.96 & / \\
\hline Method $E^{37}$ & xylan-AuNPs & $0-1000$ & 0.57 & l \\
\hline Method $\mathrm{F}^{38}$ & GSH-AuNCs & $2.49-800$ & 0.42 & I \\
\hline Method $\mathrm{G}^{39}$ & $\begin{array}{l}\text { chemical probe } \\
\text { (BODIPY-coumarin } \\
\text { fuorophores) }\end{array}$ & $0-100$ & 0.366 & l \\
\hline Method $\mathrm{H}^{40}$ & $\begin{array}{l}\text { chemical probe } \\
\text { (ACA) }\end{array}$ & $0-40$ & 0.657 & 20 \\
\hline
\end{tabular}

Note: "/" donates no clear record in the reference. 


\section{Discrimination of D-/L-Cys}

This study was conducted based on the interactions between different organic acids and different chiral Cys and realized the selective recognition of D-/L-Cys. According to previous reports, mandelic acid had an interaction with D-Cys, ${ }^{41}$ while tartaric acid had an interaction with L-Cys. ${ }^{42}$ In the present work, mandelic acid and tartaric acid were added into the GSH-AuNCs solution separately to realize the selective discrimination of D-/L-Cys. Photos under sunlight and UV light were recorded and UV and FL spectrum data were collected for further analysis. As shown in Fig. 9, when mandelic acid was added, only L-Cys caused a significant aggregation of GSH-AuNCs due to the stronger interaction between mandelic acid and D-Cys, and this change was visible under sunlight and UV light. The UV spectrum showed that the absorbance wavelength at 300-400 $\mathrm{nm}$ of the solution with L-Cys increased significantly while adding D-Cys, the absorbance of the solution did not change much, and the UV spectrum was almost monodispersed. The fluorescence spectra of the samples were collected at the excitation wavelength of $398 \mathrm{~nm}$ showed in Fig. 9(D). The fluorescence intensity of the AuNCs-GSH solution containing D-/L-Cys was increased by 1.2/2.4 times, respectively. 


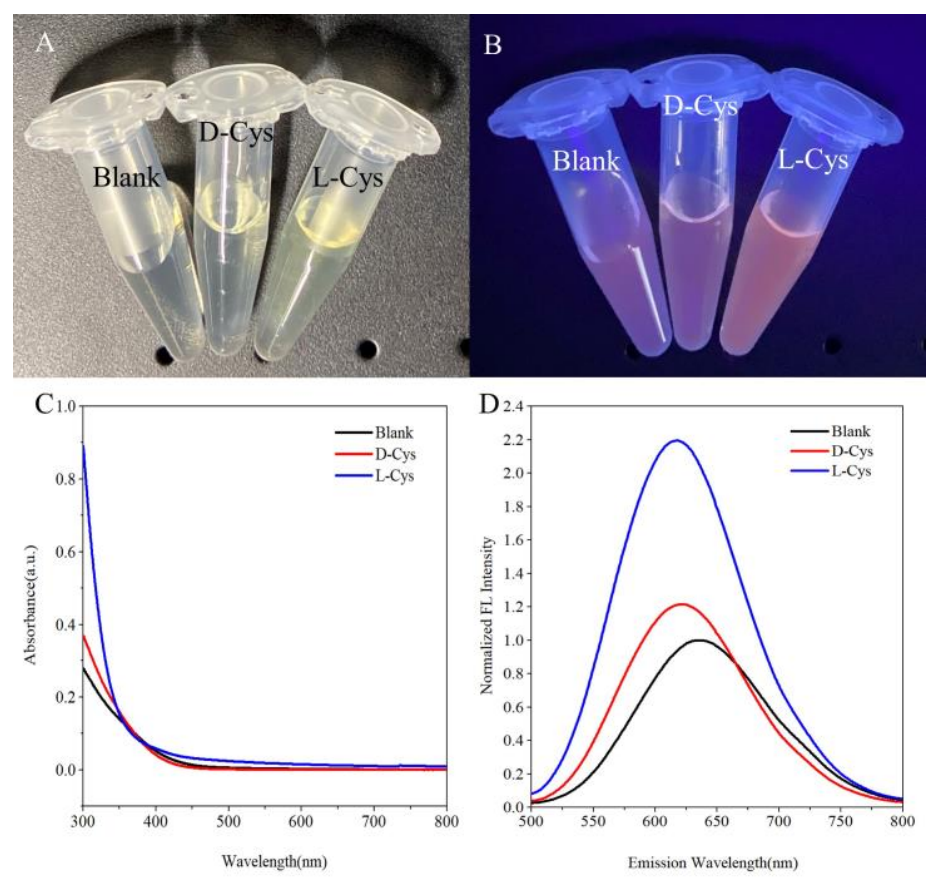

Fig. 9 Digital photographs of GSH-AuNCs/ $\mathrm{Ag}^{+}$system responding to D-/L-Cys in the mandelic acid (100 mM) under (A) visible light and (B) UV light. (C) The UV spectra of the above solutions. (D) Fluorescence spectra at $620 \mathrm{~nm}$ of D/L-Cys in mandelic acid buffer.

As expected, when tartaric acid, which tended to coordinate with L-Cys, was added, aggregation caused only by D-Cys was observed. This change could be distinguished by the naked eyes under sunlight and UV light. Similarly, the UV spectra illustrated that the absorbance of the solution with D-Cys at $300-400 \mathrm{~nm}$ was significantly enhanced, while the UV spectrum of the solution with L-Cys was almost monodisperse and slightly changed. Using $398 \mathrm{~nm}$ as excitation wavelength, fluorescence spectra were collected and showed in Fig. 10, and the fluorescence emission intensity of adding D-/L-Cys increased by 1.5 times/1.1 times. Based on the results above, D-/L-Cys were distinguished with naked eyes. Thus, the method had a promising application in chiral discrimination and resolution. 



Fig. 10 Digital photographs of GSH-AuNCs/ $\mathrm{Ag}^{+}$system responding to D-/L-Cys in the tartaric acid (100 mM) under (A) visible light and (B) UV light. (C) The UV spectra of the above solutions. (D) Fluorescence spectra at $620 \mathrm{~nm}$ of D/L-Cys in tartaric acid buffer.

\section{Conclusions}

In summary, a method for rapid determination of Cys by using GSH-AuNCs was established. Selective analysis of the enantiomer of Cys was also achieved by this method. The mechanism behind the determination was driven by the coordination between transition metal ions and GSH-AuNCs. $\mathrm{As}^{\mathrm{Ag}^{+}}$have a coordination effect with GSH-AuNCs, Cys could coordinate with $\mathrm{Ag}^{+}$to form the complex and then bridge GSH-AuNCs. A regular network structure of the complex indicated that Cys connects GSH-AuNCs with $\mathrm{Ag}^{+}$as a bridging agent. The network structure restricted intramolecular vibration and rotation and reduced intermolecular collisions. Accordingly, the energy loss caused by the non-radiative transition decreased and AIEE process 
occurred. Therefore, the concentration of Cys could be quantitatively determined depending on the variation of fluorescence intensity. More importantly, due to the large steric hindrance of GSH, fluorescence enhancement was hardly produced at the same concentration, thus improving the specificity of detection. As the AIEE effect occurred rapidly, the detection process could be completed within two minutes. Moreover, due to the specific interactions of D-/L-Cys with mandelic acid and tartaric acid, the chiral discrimination of D-/L-Cys with naked eyes was realized by replacing the organic acid buffer. The results showed that AIEE has a potential of being applied to chiral discrimination.

\section{Acknowledgements}

This research was financially supported by the National Natural Science Foundation of China (Nos. 81973285 and 81573397) and Shanghai Natural Science Foundation (15ZR1409400). 


\section{References}

1. K. Axelsson, B. Mannervik, FEBS Lett., 1983, 152, 114-118.

2. Z. A. Wood, E. S. der, J. R. Harris, and L. B. Poole, Trends Biochem. Sci., 2003, 28, $32-40$.

3. E. Weerapana, C. Wang, G. M. Simon, F. Richter, S. Khare, M. B. D. Dillon, D. A. Bachovchin, K. Mowen, D. Baker, and B. F. Cravatt, Nature., 2010, 468, 790-795.

4. C. L. Jones, B. M. Stevens, A. D’Alessandro, R. Culp-Hill, J. A. Reisz, S. Pei, A. Gustafson, N. Khan, J. DeGregori, D. A. Pollyea, and C. T. Jordan, Blood., 2019, 134, 389-394.

5. A. Badaloo, J. W. Hsu, C. Taylor-Bryan, C. Green, M. Reid, T. Forrester, and F. Jahoor, Am. J. Clin. Nutr., 2012, 95, 84-90.

6. M. D. Kornberg, P. Bhargava, P. M. Kim, V. Putluri, A. M. Snowman, N. Putluri, P. A. Calabresi, and S. H. Snyder, Science., 2018, 360, 449-453.

7. M. W. Lieberman, A. L. Wiseman, Z. Z. Shi, B. Z. Carter, R. Barrios, C. N. Ou, P. Chevez-Barrios, Y. Wang, G. M. Habib, J. C. Goodman, S. L. Huang, R. M. Lebovitz, and M. M. Matzuk, Proc. Natl. Acad. Sci. U. S. A., 1996, 93, 7923-7926.

8. L. Huang, H. Ying, Z. Chen, Y. 1. Zhu, Y. Gu, L. Hu, D. Chen, and N. Zhong, Placenta., 2019, 80, 27-35.

9. S. Chu, Z. Niu, Q. Guo, H. Bi, X. Li, F. Li, Z. Zhang, W. He, P. Cao, N. Chen, and X. Sun, Eur. J. Pharmacol., 2020, 882, 173258.

10. M. F. McCarty, J. H. O'Keefe, and J. J. DiNicolantonio, Med. Hypotheses., 2019, $132,109356$.

11. Y. KoSUGE, Exp. Ther. Med., 2020, 19, 1565-1569.

12. A. Slivka, G. Cohen, Brain Res., 1993, 608, 33-37.

13. N. Shibuya, S. Koike, M. Tanaka, M. Ishigami-Yuasa, Y. Kimura, Y. Ogasawara, K. Fukui, N. Nagahara, and H. Kimura, Nat. Commun., 2013, 4, S26-S26. 
14. C. H. Misra, Neurochem. Res., 1989, 14, 253-257.

15. H. Peng, Z. Huang, H. Deng, W. Wu, K. Huang, Z. Li, W. Chen, and J. Liu, Angew. Chem. Int. Ed. Engl., 2020, 59, 9982-9985.

16. Z. T. Luo, X. Yuan, Y. Yu, Q. B. Zhang, D. T. Leong, J. Y. Lee, and J. P. Xie, J. Am. Chem. Soc., 2012, 134, 16662-16670.

17. Z. T. Luo, V. Nachammai, B. Zhang, N. Yan, D. T. Leong, D. E. Jiang, and J. P. Xie, J. Am. Chem. Soc., 2014, 136, 10577-10580.

18. T. Shu, L. Su, J. Wang, X. Lu, F. Liang, C. Li, and X. Zhang, Anal. Chem., 2016, 88, 6071-6077.

19. Y. Guo, X. Tong, L. Ji, Z. Wang, H. Wang, J. Hu, and R. Pei, Chem. Commun., 2015, 51, 596-598.

20. N. Murshid, D. S. Smith, and V. Kitaev, Part. Part. Syst. Charact., 2018, 35, 1800285.

21. J. Luo, Z. Xie, J. W. Y. Lam, L. Cheng, H. Chen, C. Qiu, H. S. Kwok, X. Zhan, Y. Liu, D. Zhu, and B. Z. Tang, Chem. Commun., 2001, 18, 1740-1741.

22. Y. Yu, J. Li, T. Chen, Y. N. Tan, and J. Xie, J. Phys. Chem. C., 2015, 119, 10910-10918.

23. Z. Gan, Y. Lin, L. Luo, G. Han, W. Liu, Z. Liu, C. Yao, L. Weng, L. Liao, J. Chen, X. Liu, Y. Luo, C. Wang, S. Wei, and Z. Wu, Angew. Chem. Int. Ed. Engl., 2016, 55, $11567-11571$.

24. Y. Wei, W. Luan, F. Gao, and X. Hou, Part. Part. Syst. Charact., 2019, 36, 1900314.

25. Z. Wu, H. Liu, T. Li, J. Liu, J. Yin, O. F. Mohammed, O. M. Bakr, Y. Liu, B. Yang, and H. Zhang, J. Am. Chem. Soc., 2017, 139, 4318-4321.

26. W. X. Wang, Y. Wu, and H. W. Li, J. Colloid Interface Sci., 2017, 505, 577-584.

27. Q. Lai, Q. Liu, K. Zhao, X. Duan, G. Wang, and X. Su, Microchim. Acta., 2019, 186, 327. 
28. M. Wu, J. Zhao, D. M. Chevrier, P. Zhang, and L. Liu, J. Phys. Chem. C., 2019, 123, 6010-6017.

29. X. Lai, L. Tan, X. Deng, J. Liu, A. Li, J. Liu, and J. Hu, ACS. Appl. Mater. Inter., $2017,9,5118-5127$.

30. J. Liu, H. Liu, Y. Li, and H. Wang, J. Biol. Phys., 2014, 40, 313-323.

31. W. Ding, Y. Liu, Y. Li, Q. Shi, H. Li, H. Xia, D. Wang, and X. Tao, RSC. Adv., 2014, 4, 22651-22659.

32. X. Jiang, J. Huang, T. Chen, Q. Zhao, FengXu, and X. Zhang, Int. J. Biol. Macromol., 2020, 153, 412-420.

33. N. W. Pirie, K. G. Pinhey, J. Biol. Inorg. Chem., 1929, 84, 321-333.

34. S. Chen, Y. Luo, N. Wang, X. Chen, Y. Guo, H. Deng, J. Xu, S. W. Chen, and J. Wang, Talanta., 2020, 208, 119934.

35. X. Yang, Y. Wang, M. X. Zhao, and W. Yang, Spectrochim. Acta. A. Mol. Biomol. Spectrosc., 2019, 212, 10-14.

36. S. Zhang, D. Wu, J. Wu, Q. Xia, X. Jia, X. Song, L. Zeng, and Y. Yuan, Talanta., 2019, 204, 747-752.

37. Y. Luo, Z. Shen, P. Liu, L. Zhao, and X. Wang, Carbohydr. Polym., 2016, 140, $122-128$

38. Y. Zhang, H. Xu, Y. Chen, X. You, Y. Pu, W. Xu, and X. Liao, J. Fluoresc., 2020, 30, 1491-1498.

39. X. L. Liu, L. Y. Niu, Y. Z. Chen, Y. Yang, and Q. Z. Yang, Biosens. Bioelectron., 2017, 90, 403-409.

40. X. Dai, Q. H. Wu, P. C. Wang, J. Tian, YuXu, S. Q. Wang, J. Y. Miao, and B. X. Zhao, Biosens. Bioelectron., 2014, 59, 35-39.

41. H. Huang, L. Hu, Y. Sun, Y. Liu, Z. Kang, and D. R. MacFarlane, Mikrochim. Acta., 2019, 186, 298. 
42. G. Song, C. Xu, and B. Li, Sens. Actuators B., 2015, 215, 504-509. 